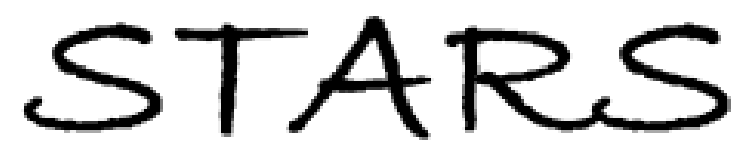

University of Central Florida

STARS

$1-1-2002$

\title{
Second-harmonic generation tuning curves with narrow, high- intensity beams for quasiphase-matched potassium titanyl phosphate
}

Hongki Kim

University of Central Florida

Ladislav Jankovic

University of Central Florida

George Stegeman

University of Central Florida

Mordechai Katz

Silvia Carrasco

Find similar works at: https://stars.library.ucf.edu/facultybib2000

University of Central Florida Libraries http://library.ucf.edu See next page for additional authors

This Article is brought to you for free and open access by the Faculty Bibliography at STARS. It has been accepted for inclusion in Faculty Bibliography 2000s by an authorized administrator of STARS. For more information, please contact STARS@ucf.edu.

\section{Recommended Citation}

Kim, Hongki; Jankovic, Ladislav; Stegeman, George; Katz, Mordechai; Carrasco, Silvia; and Torner, Lluis, "Second-harmonic generation tuning curves with narrow, high-intensity beams for quasiphase-matched potassium titanyl phosphate" (2002). Faculty Bibliography 2000s. 3288.

https://stars.library.ucf.edu/facultybib2000/3288

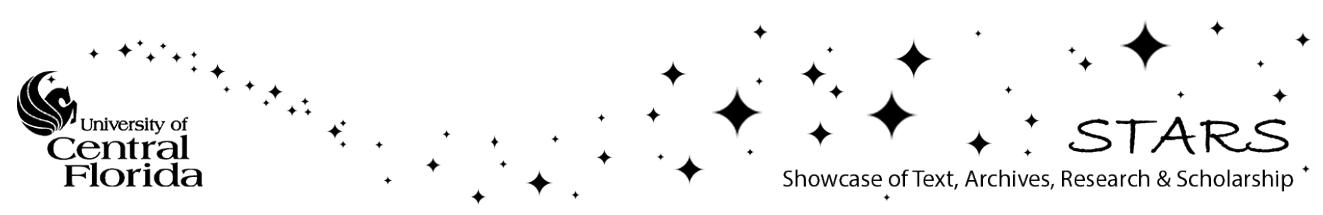


Authors

Hongki Kim, Ladislav Jankovic, George Stegeman, Mordechai Katz, Silvia Carrasco, and Lluis Torner 


\section{Second-harmonic generation tuning curves with narrow, high-intensity beams for quasiphase-matched potassium titanyl phosphate}

Cite as: Appl. Phys. Lett. 81, 2710 (2002); https://doi.org/10.1063/1.1512941

Submitted: 24 June 2002 . Accepted: 16 August 2002 . Published Online: 30 September 2002

Hongki Kim, Ladislav Jankovic, George Stegeman, Mordechai Katz, Silvia Carrasco, and Lluis Torner

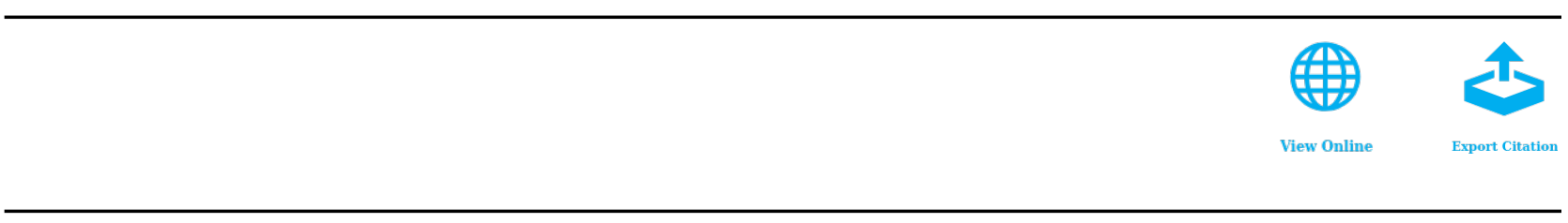

Applied Physics Letters

Mid-IR and THz frequency combs special collection 


\title{
Second-harmonic generation tuning curves with narrow, high-intensity beams for quasiphase-matched potassium titanyl phosphate
}

\author{
Hongki Kim, ${ }^{\text {a) }}$ Ladislav Jankovic, and George Stegeman \\ CREOL/School of Optics, University of Central Florida, 4000 Central Florida Boulevard, Orlando, \\ Florida 32816 \\ Mordechai Katz \\ Electro-Optic Div., Soreq NRC, Yavene, 81800, Israel \\ Silvia Carrasco and Lluis Torner \\ Universitat Politecnica de Catalunya, Departamento of Signal Theory and Communications, \\ 08034 Barcelona, Spain
}

(Received 24 June 2002; accepted 16 August 2002)

\begin{abstract}
The tuning curves with temperature and incidence angle for second-harmonic generation were asymmetrically distorted and broadened with increasing intensity for narrow input fundamental beams in periodically poled $\mathrm{KTiOPO}_{4}$. Multiple phenomena including mutual self-focusing of the fundamental and harmonic, cascading, quadratic soliton generation, and artificially induced walk-off (for light incidence away from the poling axis) contribute, in good agreement with theory. (C) 2002 American Institute of Physics. [DOI: 10.1063/1.1512941]
\end{abstract}

Second harmonic $(\mathrm{SH})$ generation $(\mathrm{SHG})$ has become a very versatile techniques for extending the frequency range of lasers. ${ }^{1}$ It has been enhanced by the invention of quasiphase-matched (QPM) periodically poled (PP) structures which allow noncritical phasematching (NCPM) for SHG at virtually any wavelength. ${ }^{2}$ Very efficient SHG has been reported in bulk $\mathrm{PP}-\mathrm{LiNbO}_{3}$ (PPLN) and $\mathrm{PP}-$ potassium titanyl phosphate (PPKTP), see Refs. 3 and 4. Furthermore, the distortion of the SHG tuning curves, predicted for NCPM for narrow beams with broad distributions in incident wave vector, ${ }^{5}$ has been reported. ${ }^{6,7}$ However, at high enough intensities, second order nonlinear effects such as mutual beam narrowing and cascading of the fundamental (FW) and harmonic produce additional beam distortions, leading under appropriate conditions to the generation of quadratic spatial solitons (QSSs). ${ }^{8,9}$ QSSs have been reported in PPLN, with an intensity threshold of a few $\mathrm{GW} / \mathrm{cm}^{2}$ for beam waists of tens of microns. ${ }^{10}$ Here we show that all of these phenomena have a large impact on SHG tuning curves in NCPM PPKTP for narrow, high intensity input beams.

The equations associated with the interaction between a FW $\left\{a_{1}(y, z) \exp \left[i\left(\omega t-k_{1} x\right)\right]\right\}$ beam and it is copolarized $\mathrm{SH}$ $\left\{a_{2}(y, z) \exp \left[i\left(2 \omega t-k_{2} x\right)\right]\right\}$ near phase matching are well known. For general light propagation they can be written as

$i \frac{\partial a_{1}}{\partial x}-\frac{1}{2 k_{1}}\left(\frac{\partial^{2}}{\partial y^{2}}+\frac{\partial^{2}}{\partial z^{2}}\right) a_{1}+\Gamma(x, y) a_{1}^{*} a_{2} \exp (-i \Delta k x)=0$,

$i \frac{\partial a_{2}}{\partial x}-\frac{1}{2 k_{2}}\left(\frac{\partial^{2}}{\partial y^{2}}+\frac{\partial^{2}}{\partial z^{2}}\right) a_{2}+\Gamma(x, y) a_{1}^{2} \exp (i \Delta k x)=0$,

where $\Delta k=2 k_{1}-k_{2}, \Gamma(x, y)$ changes sign periodically along the poling $x$ axis with period $\Lambda=2 \pi / \kappa,|\Gamma| \propto d_{\text {eff }}^{(2)}$, and $d_{\mathrm{eff}}^{(2)}=2 d_{33}^{(2)} / \pi$ for PPKTP. For propagation along the poling axis, the phase mismatch is $\Delta \varphi=2 \omega\left(n_{1}-n_{2}\right.$

${ }^{a)}$ Electronic mail: hongki@creol.ucf.edu $+\pi c / \Lambda \omega) L / c$. If the QPM contribution is added to the fundamental's refractive index surface, the cuts of the index surfaces for phase matching in the $x-y$ plane are shown in Fig. 1. For off $x$ axis incidence, an artificially induced "walk-off" of the FW from the SH occurs at an angle $\psi$ given by $\sin \psi=\sin \theta \kappa / k_{2}$ for internal incidence angle $\theta$ from the $x$ axis. ${ }^{2,11}$ This extra walk-off is the main difference between QPM and birefringence NCPM.

Solving these coupled wave equations yields the detailed dynamics of the interacting beams and the output SHG conversion efficiency. In the absence of diffraction the spatial profile of the $\mathrm{SH}$ is proportional to $a_{1}^{2}$ and therefore is narrower in space than the FW. ${ }^{9}$ Similarly, downconversion by which photons return to the $\mathrm{FW}$ from the $\mathrm{SH}$ is proportional to $a_{2} a_{1}^{*}$ so that the regenerated $\mathrm{FW}$ is narrower than the original FW. 9 In addition, "cascading" produces nonlinear phase shifts that vary across the beam due to the differential phase velocity between the FW and SH off phase match and
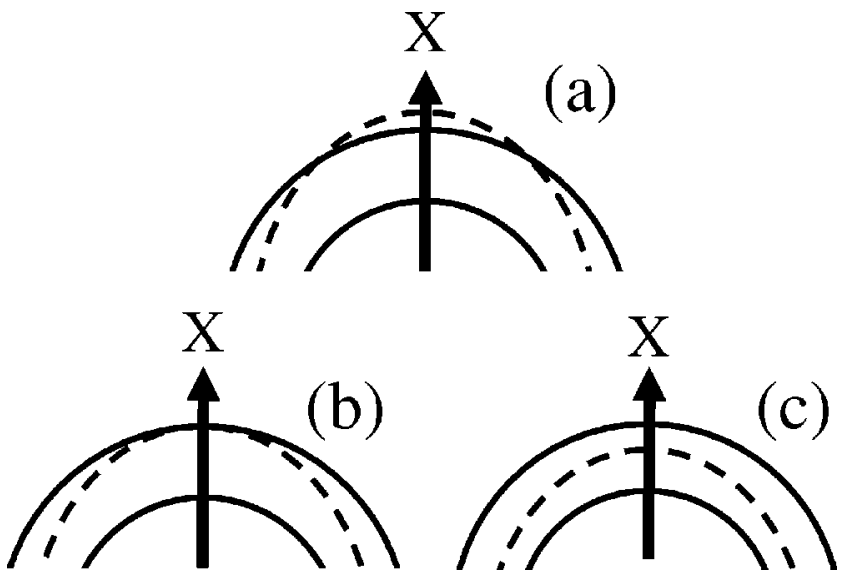

FIG. 1. Cuts of the index ellipsoids in the $x-y$ plane for $z$-polarized FW (inside solid lines) and SH (outside solid lines). The dashed line simulates approximately the effect of the nonlinear QPM grating. (a) $T<T_{\mathrm{PM}}(\Delta k$ $>0)$; (b) $T=T_{\mathrm{PM}}(\Delta k=0)$; (c) $T>T_{\mathrm{PM}}(\Delta k<0)$. 

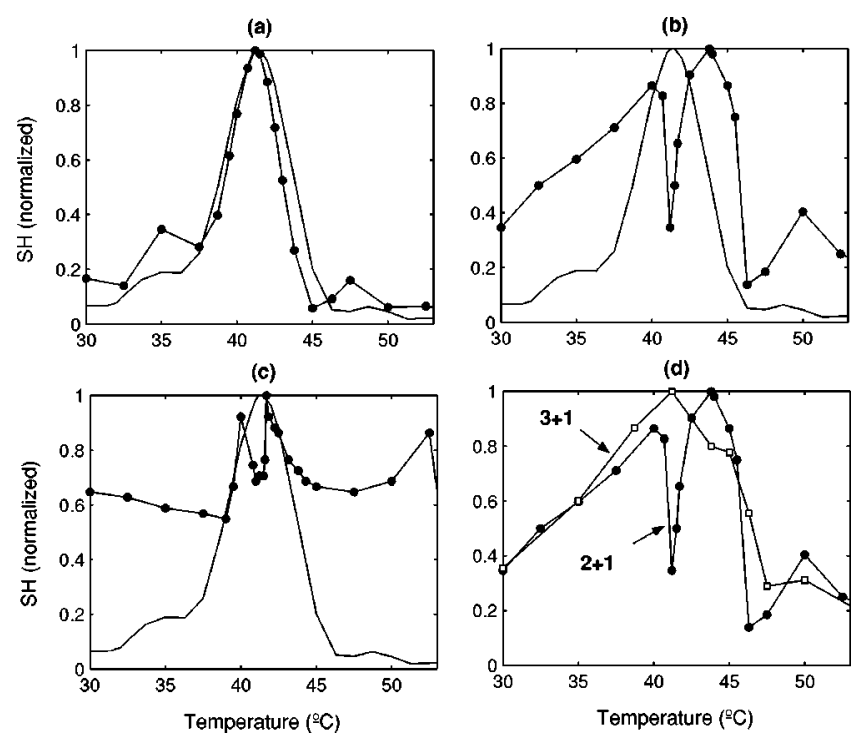

FIG. 2. Calculated SHG tuning curves for temperature detuning from phase match for an $18 \mu \mathrm{m}$ input beam waist and a crystal length of $1 \mathrm{~cm}$. Solid lines: cw simulations for a low intensity, $\sim 0.22 \mathrm{MW} / \mathrm{cm}^{2}$. 0 : cw simulations for input intensities of (a) $\sim 88$, (b) $\sim 550$, and (c) $\sim 2.6 \mathrm{GW} / \mathrm{cm}^{2}$. In (d), a full $3+1$ spatiotemporal simulation is shown for a peak input intensity of $\sim 550 \mathrm{MW} / \mathrm{cm}^{2}$ and $20 \mathrm{ps}$ pulses.

also contributes to beam focusing. ${ }^{9}$ Such contributions are different for $\Delta k>0$, and $\Delta k<0$. It is this beam dynamics that deviates the high-power SHG tuning curves from their well-known low power predictions.

The generation of QSSs, a stationary balance between diffraction and beam narrowing also affects the SHG tuning curves. Soliton fields exhibit different properties from those of standard SHG. For efficient SHG on phase match the fields are $\pi / 2$ out of phase and energy flows from the FW into the SH with distance. Off phase match, the relative phase between the fields rotates with distance and there is a periodic energy exchange between the fields. In contrast to this, the two soliton components, $a_{1}$ and $a_{2}$, have both constant amplitude and relative phase with distance, irrespective of the initial $\Delta k$. Once the soliton is formed no further $\mathrm{SH}$ is generated. Almost all soliton generation experiments to date have had FW only inputs and initially the SH field grew out of phase with the FW. These fields evolve nonadiabatically over a few parametric gain lengths $\left[l_{\mathrm{pg}}=\left|\Gamma a_{1}(0)\right|^{-1}\right]$ into a soliton and the excess FW and SH energy is radiated away. In this limit, the SHG efficiency depends on the energy sharing soliton properties, ${ }^{12}$ the radiation fields generated and the sample length plays no role. ${ }^{13,14}$

Because full $(3+1) D$ spatiotemporal simulations are very time consuming, and because the pulses are too long to produce group velocity mismatch problems between the fundamental and harmonic, most of the numerical simulations were performed for $\mathrm{cw}$ excitation in order to identify the essential physics associated with high intensity, narrow beam inputs. Numerical results for the cw SHG tuning curves on NCPM phase match [Fig. 1(b)] were obtained for increasing input intensity, Figs. 2(a)-2(c). In Fig. 2(d), an example of full $(3+1)$ spatiotemporal simulation is shown for comparison with the $(2+1)$ case. The gross $\mathrm{cw}$ tuning features with deep minima and maxima are "averaged" out so in general one would not expect sharp maxima or minima to occur with
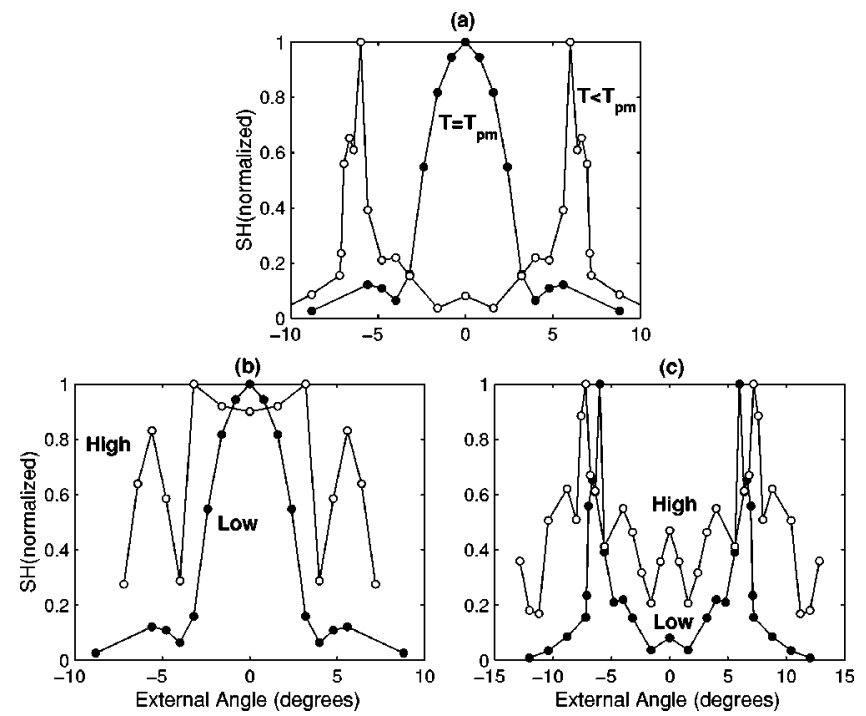

FIG. 3. Calculated angle tuning SHG for cw excitation of an input beam of $18 \mu \mathrm{m}$ and a crystal length of $1 \mathrm{~cm}$. In (a) low input intensity, $\sim 90 \mathrm{~kW} / \mathrm{cm}^{2} ;-T\left(42.7^{\circ} \mathrm{C}\right)=T_{\mathrm{PM}}$ and $\bigcirc-T\left(30.0^{\circ} \mathrm{C}\right)<T_{\mathrm{PM}}$. In (b) $T\left(42.7^{\circ} \mathrm{C}\right)=T_{\mathrm{PM}} ;-$ low input intensity, $\sim 90 \mathrm{~kW} / \mathrm{cm}^{2}$ and $\bigcirc$-high powers of $\sim 225 \mathrm{MW} / \mathrm{cm}^{2}$. In (c), $T\left(30.0^{\circ} \mathrm{C}\right)<T_{\mathrm{PM}} ;-$-low intensities $\sim 90 \mathrm{~kW} / \mathrm{cm}^{2}$ and $\bigcirc$-high intensities $\sim 1 \mathrm{GW} / \mathrm{cm}^{2}$.

pulsed excitation. The response curves broaden with increasing intensity, taking on multipeak structures far from the classical plane wave and narrow beam modified $\operatorname{sinc}^{2}$ response. This occurs because $l_{\mathrm{pg}}$ decreases with increasing input intensity. At very high intensities, the response curves for $\Delta k<0$ exhibit a great deal of detailed structure which changes rapidly with increasing intensity because of strong competition between beam narrowing and cascading. cw numerical results for angle tuning in the geometry of Fig. 1(a) are shown in Fig. 3, for different temperatures and input powers. Although the tuning curves remain symmetric about the poling axis, the two phase-matching peaks broaden asymmetrically with increasing power for the reasons discussed previously so that SHG in the region between the peaks rises dramatically.

The PPKTP samples were fabricated using the low tem-

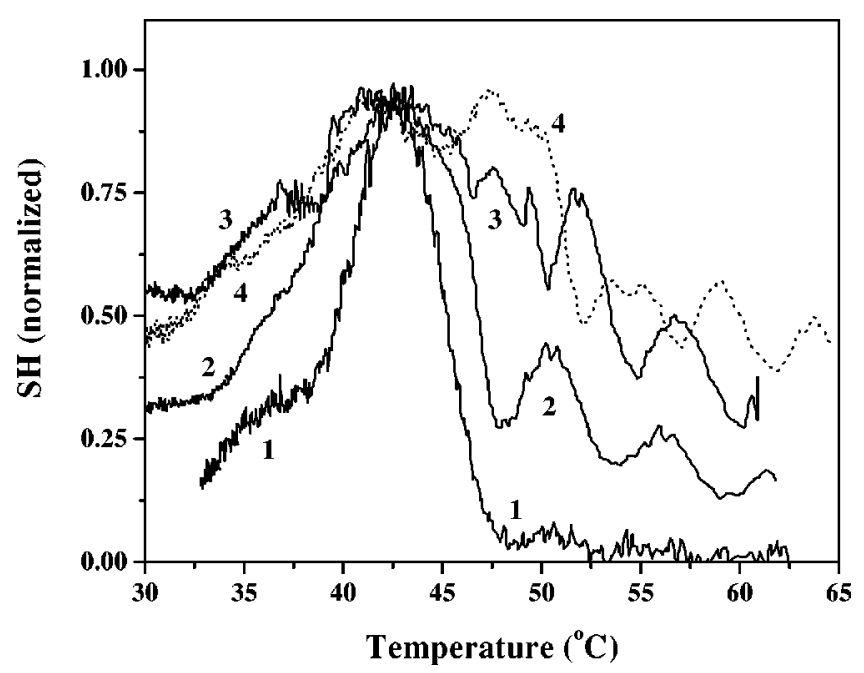

FIG. 4. Observed tuning curves for SHG obtained by temperature tuning around the low intensity phase-match temperature $\left(42.7^{\circ} \mathrm{C}\right) .1-0.02$, 2-2.0, 3-6.1, 4-12.0 GW/ $/ \mathrm{cm}^{2}$. 


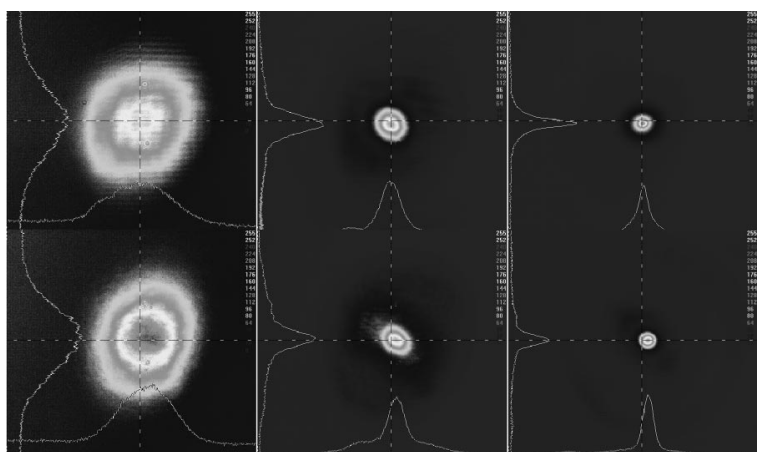

$0.5 \mathrm{GW} / \mathrm{cm}^{2}$

$2 \mathrm{GW} / \mathrm{cm}^{2}$

$6.1 \mathrm{GW} / \mathrm{cm}^{2}$

FIG. 5. Fundamental (upper) and second harmonic (lower) output beam profiles for different input intensities on phase match.

perature periodic poling which gave a $d_{\text {eff }}\left(=2 d_{33} / \pi\right)$ of 9.5 $\mathrm{pm} / \mathrm{V} .^{15}$ For $\Lambda=8.99 \mu \mathrm{m}$, phase matching occurred at $42.7^{\circ} \mathrm{C}$. The $1 \mathrm{~cm}$ long sample had a previously measured low-power SHG bandwidth [full width at half maximum (FWHM)] of $0.2 \mathrm{~nm}$, in good agreement with theory, indicating that phase matching is realized over the full length. ${ }^{15}$ An EKSMA Nd:YAG laser-amplifier with 25 ps width at 10 $\mathrm{Hz}$ provided a highly symmetric laser beam that was improved further by spatial filtering to give $M^{2}$ of 1.1-1.2 with a FWHM bandwidth of $0.11-0.14 \mathrm{~nm}$, sufficient to resolve the low intensity SHG side lobes for broad input beams. The $z$-polarized $1064 \mathrm{~nm}$ beam was focused by lenses to a minimum beam waist $w_{0}=16.4 \mu \mathrm{m}$ as measured by the knifeedge method, giving 7.5 diffraction lengths of propagation along the $x$ axis.

The experimental results for temperature tuning with $x$ axis incidence are shown in Fig. 4. As predicted, massive broadening and distortion occurs in the tuning curves at high intensities. On phase match the energy conversion of the FW input into $\mathrm{SH}$ peaks at $48 \%$ around $2 \mathrm{GW} / \mathrm{cm}^{2}$ of $\mathrm{FW}$ intensity, and then decreases with input intensity to $1 / 2$ of the peak value at $20 \mathrm{GW} / \mathrm{cm}^{2}$. The output beam profiles in Fig. 5 show strong narrowing of both the FW and SH around a few $\mathrm{GW} / \mathrm{cm}^{2}$, coincident with maximum SHG. At lower input intensities (not shown), the energy oscillates between the FW and $\mathrm{SH}$ due to successive up- and downconversion cycles. ${ }^{9}$ Single soliton generation occurs around $3.5 \mathrm{GW} / \mathrm{cm}^{2}$ and solitons dominate the output profile for further increase in intensity. Once solitons are formed, the fraction of SH in the soliton becomes constant with distance. Increasing the input intensity leads both to an increase in the SH content of the soliton, ${ }^{12-14}$ and a smaller conversion of the input into the soliton energy due to increased radiation losses with the latter dominating the SHG efficiency.

The output beam profiles for $T<T_{\mathrm{PM}}$ resemble those at phase-match because the contribution due to cascading augments the beam narrowing process for $\Delta k>0$. For $T>T_{\mathrm{PM}}$ $(\Delta k<0)$ the soliton threshold rises rapidly with detuning. ${ }^{13,14}$ The output beams shown in Fig. $6(\Delta k$ $=-2 \pi \mathrm{cm}^{-1}$ ) contain no solitons and exhibit complicated shapes due to the combination of up- and downconversion, cascading, and beam narrowing which lead to the oscillations in the response curves.

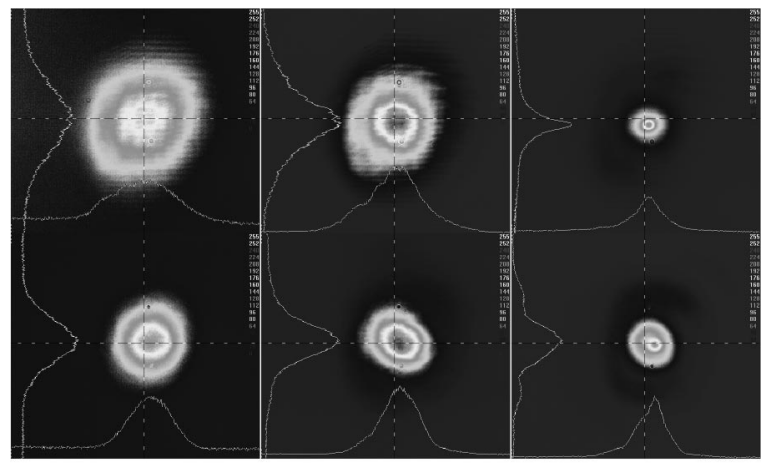

$0.5 \mathrm{GW} / \mathrm{cm}^{2}$

$2 \mathrm{GW} / \mathrm{cm}^{2}$

$6.1 \mathrm{GW} / \mathrm{cm}^{2}$

FIG. 6. Fundamental (upper) and second harmonic (lower) output beam profiles for different input intensities at $47.7^{\circ} \mathrm{C}, T>T_{\mathrm{PM}}(\Delta k=-2 \pi$ $\left.\mathrm{cm}^{-1}\right)$.

In summary, the SHG tuning curves in PPKTP have been studied with narrow input beams at high input intensities. The largest impact on SHG was the mutual beam narrowing of the FW and SH. On phase match, the SHG efficiency peaks when beam narrowing is strong and at higher intensities the SHG efficiency decreases with input intensity, a result probably due to the increased radiation losses and high two-photon absorption exhibited by KTP at harmonic intensities $>10 \mathrm{GW} / \mathrm{cm}^{2}$. The detuning curves exhibit large intensity-dependent broadening and intensity-dependent asymmetry between the $\Delta k>0$ and $\Delta k<0$ sides due to cascading.

The US research was supported by an ARO MURI, the US-Spanish collaboration by the Commission for Scientific Exchange between the USA and Spain, and by the Spanish Government through TIC2000-1010. H. Kim gratefully acknowledges the support of the postdoctoral fellowship program from the Korea Science \& Engineering Foundation (KOSEF).

${ }^{1}$ C. L. Tang, W. R. Bosenberg, T. Ukachi, R. J. Lane, and L. K. Cheng, Proc. IEEE 80, 365 (1992).

${ }^{2}$ Reviewed in M. M. Fejer, G. A. Magel, D. H. Jundt, and R. L. Byer, IEEE J. Quantum Electron. 28, 2631 (1992).

K. R. Parameswaran, J. R. Kurz, R. V. Roussev, and M. M. Fejer, Opt. Lett. 27, 43 (2002).

${ }^{4}$ A. Englander, R. Lavi, M. Katz, M. Oron, D. Eger, E. Lebiush, G. Rosenman, and A. Skliar, Opt. Lett. 22, 1598 (1997).

${ }^{5}$ G. D. Boyd and D. A. Kleinman, J. Appl. Phys. 39, 3597 (1965).

${ }^{6}$ G. M. Gibson, G. A. Turnbull, M. Ebrahimzadeh, M. H. Dunn, H. Karlsson, G. Arvidsson, and F. Laurell, Appl. Phys. B: Lasers Opt. 67, 675 (1998).

K. Fradkin-Kashi, A. Arie, A. Skliar, and G. Rosenman, Appl. Phys. Lett. 74, 914 (1998).

${ }^{8}$ Yu. N. Karamzin and A. P. Sukhorukov, Zh. Eksp. Teor. Fiz. 68, 834 (1975) [Sov. Phys. JETP 41, 414 (1976)]

${ }^{9}$ G. I. Stegeman, D. J. Hagan, and L. Torner, Opt. Quantum Electron. 28, 1691 (1996)

${ }^{10}$ B. Bourliaguet, V. Couderc, A. Barthelemy, G. W. Ross, P. G. R. Smith, D. C. Hanna, and C. De Angelis, Opt. Lett. 24, 1410 (1999).

${ }^{11}$ C. B. Clausen and L. Torner, Opt. Lett. 24, 7 (1999).

${ }^{12}$ L. Torner, Opt. Commun. 114, 136 (1995).

${ }^{13}$ R. A. Fuerst, M. T. G. Canva, D. Baboiu, and G. I. Stegeman, Opt. Lett. 22, 1748 (1997).

${ }^{14}$ L. Torner, J. P. Torres, D. Artigas, D. Mihalache, and D. Mazilu, Opt. Commun. 164, 153 (1999).

${ }^{15}$ A. Arie, G. Rosenman, A. Korenfield, A. Skliar, M. Oron, M. Katz, and D. Eger, Opt. Lett. 23, 28 (1998). 